\title{
Experiencias migratorias de los varones homosexuales y bisexuales colombianos en España
}

\section{Gayand bisexual Colombian men's migratoryexperiencesinSpain}

\author{
* Jair Eduardo Restrepo Pineda \\ Corporación Universitaria Minuto de Dios-UNIMINUTO; Sede Bello. Facultad de Ciencias Humanas y \\ Sociales, Programa de Trabajo Social. Colombia. \\ jair.restrepo@uniminuto.edu
}

Recibido / Received: 18/03/2016

Aceptado / Accepted: 11/09/2016

\section{RESUMEN}

El presente artículo tiene como objetivo visibilizar el papel de la identidad sexual en la motivación y consolidación del proceso migratorio de los varones homosexuales y bisexuales colombianos que residen en España. Para tal fin, se emplea una metodología cualitativa basada en la realización de 34 entrevistas en profundidad a igual número de hombres que residen en diferentes ciudades españolas. Se pudo constatar que en algunos casos, la identidad sexual aparece como un factor determinante para dar inicio a la migración y en otros se relaciona con aspectos personales, familiares y sociales tanto del país de origen como de destino que pueden consolidar dicho proceso. Sin embargo, se hizo evidente que ciertos proyectos migratorios estaban justificados bajo otro tipo de motivaciones que finalmente eran parte integral de un proceso migratorio estructurado en torno a la identidad sexual de quien migra.

Palabras clave: Migración, Sexualidad, Homosexualidad, Bisexualidad, Identidad.

\section{ABSTRACT}

The aim of this article is to visualise the role of sexual identity on the motivation and consolidation of the migratory process of gay and bisexual men who live in Spain. For this purpose, a qualitative methodology has been used, based on the carrying out of 34 deep interviews to the same amount of men living in different Spanish cities. It has been established that, in some cases, sexual identity appears as a decisive factor so as to start the migration and, in some others, it is related to personal, social and family aspects from the country of origin as well as from the country of destination. These aspects can consolidate the aforementioned migratory process. Nevertheless, it has been made obvious that certain migratory projects were justified under other sort of motivations, which were finally an integral part of a migratory process structured around the migrant's sexual identity.

Keywords: Migration, Sexuality, Homosexuality, Bisexual, Identity.

\footnotetext{
*Autor para correspondencia / Corresponding author: Jair Eduardo Restrepo Pineda. Corporación Universitaria Minuto de Dios-UNIMINUTO, Sede Bello. Centro de Investigaciones, Bloque 5 Oficina 400. Carrera 45, n. ${ }^{\circ} 22$ D - 25, Bello, Antioquia, Colombia.

Sugerencia de cita / Suggested citation: Restrepo Pineda, J. E. (2017). Experiencias migratorias de los varones homosexuales y bisexuales colombianos en España. Revista Española de Sociología, 26 (2), 201-216.
} 


\section{INTRODUCCIÓN}

Un estudio sociológico de los procesos migratorios debe tener en cuenta los factores económicos, culturales y políticos, además de las condiciones de vida de los migrantes, tanto en el lugar de origen como en el de destino, así como sus motivaciones para iniciar la migración. De la misma manera, deben considerarse aspectos relacionados con la identidad sexual y de género, los cuales pueden ser parte fundamental de las causas y motivaciones para emprender los procesos migratorios, además podrán determinar las formas en las cuales los migrantes se integran en el país de destino.

La migración es definida como cualquier cambio permanente de residencia que implica "la interrupción de las actividades en un lugar y su reorganización en otro" (Goldscheider, 1971, p. 64, citado en Weeks, 1990, p. 192); sin embargo, el proceso migratorio es complejo, y para su estudio deben considerarse un conjunto de variables de los países de origen y destino, así como tiempos, trayectos e involucrados.

Para García (2013) la migración entendida desde la teoría en red es una estructura sostenida por un sistema de lazos interpersonales que conectan a los migrantes con sus antecesores, así como con los no migrantes en los países de origen y de destino. Dichas redes se basan en los vínculos de parentesco, amistad 0 pertenencia. Estas redes potencian las posibilidades de migración ya que disminuyen los costos y los riesgos del proceso y aumentan los beneficios económicos del desplazamiento; así, "cada nuevo emigrante expande la red y reduce los riesgos de desplazamiento para todos aquellos con los que está relacionado, eventualmente haciéndolos virtualmente seguros y livianamente costosos diversificando las cuotas laborales de la familia a través de la inmigración" (Massey et al., 2008, p. 459).

Por su parte, la identidad sexual se puede definir como el autoconcepto que tiene cada persona $y$ en el cual se interrelacionan el sexo, el género, la orientación sexual, la identidad de género y todo el conjunto de habilidades con las que las personas se desenvuelven en su vida cotidiana y en relación con la sexualidad. Según lo anterior, existen dife- rentes maneras de organizar la identidad sexual, y estas han variado con el transcurso de la historia y las culturas en las que se han desarrollado.

Se pueden abordar las siguientes identidades minoritarias con relación a los y las heterosexuales, considerando a estos últimos como aquellas personas que se sienten atraídas más 0 menos permanente, sexual 0 afectivamente por otras personas del sexo contrario. Así, la identidad gay "es la identificación que un hombre hace respecto de sí y comprende aspectos de la personalidad y la cultura. La sexualidad relativa a un varón gay incluye su sexo, género, orientación sexual y su capacidad para desenvolverse socialmente conforme a la percepción que tiene de sus propias capacidades sexuales". (Gallardo y Escolano, 2009, p. 27).

Por otra parte, Gallardo y Escolano (2009) definen la identidad bisexual como la identificación que un hombre 0 una mujer hacen de sí mismos por tener la capacidad de sentir atracción sexual 0 amor tanto por hombres como por mujeres. Esto no indica que desee a todo el mundo, sino que en un momento puede experimentar deseo 0 amor por un hombre $y$, en otro, por una mujer (Derechos humanos y diversidad afectiva sexual. Amnistía Internacional, 2008).También se incluyen aspectos culturales y de personalidad. Sin embargo, esta identidad ha sido invisivilizada por la sociedad.

Producto de la migración, los comportamientos pueden variar como respuesta a un nuevo contexto económico, político, social y cultural, que posibilita nuevas formas de ver y entender la identidad sexual y el género; así, dichos contextos influyen en la forma cómo se construye y asume la identidad sexual y de género y cómo cambian estos a través del proceso migratorio.

№ obstante, como los sostienen algunos autores los motivos migratorios vinculados a las identidades de género y la diversidad sexual no han sido abordados suficientemente, así "cuestiones como la violencia, el narcotráfico, las crisis económicas 0 las redes migratorias que han sido causa del estudio de la migración internacional de las últimas décadas, pocas veces han tenido una articulación desde el género, en especial con aquel ligado a la diversidad sexual, el cual no ha sido parte de la retórica nacional y regional de los desplazamientos 
de los cuerpos nacionales hacia sitios de mayor riqueza económica" (Falconí, 2015, p. 31).

De tal forma, la migración ha sido estudiada en el marco de la heteronormatividad, entendida esta como "la tendencia, en el sistema occidental contemporáneo referente al sexo/género, de considerar las relaciones heterosexuales como la norma, y todas las otras formas de conducta sexual, como desviaciones de esa norma" (Sparg0, 2007, p. 86).

La heteronormatividad establece y promueve una serie de normas relativas a la conducta y al ser que deben responder a los lineamientos que la heterosexualidad impone y sobre los cuales se fundan las diferencias de sexo y poder. Si bien lo normal se puede entender desde el punto de vista estadístico, las normas no, pues estas están establecidas moralmente y tienen la fuerza de imperativos; en consecuencia, "la heterosexualidad es "normal" en términos de estadística, pero la normatividad de las interpretaciones vigentes del sexo le garantizan el estatuto de una norma, definida en oposición a las prácticas y deseos a-normales" (Spargo, 2007, p. 86). Es así como la heteronormatividad no permite una comprensión más amplia del género y, por lo tanto, de la sexualidad, limitándola a las relaciones que se establecen entre hombres y mujeres y que responden a los criterios culturalmente construidos alrededor de esta "normalidad" del ser.

Aunque los determinantes sociales y culturales que establece la heteronormatividad están presentes en España, también se han producido en los últimos años avances legales y sociales con respecto al reconocimiento de los derechos del colectivo de lesbianas, gais, bisexuales, transexuales e intersexuales (LGBTI). Entre estos avances se encuentra la aprobación de la ley que modifica el código civil (2 de julio de 2005), permitiendo el matrimonio entre personas del mismo sexo y reconociendo, por tanto, sus derechos a la adopción conjunta, la pensión y la herencia. Simultáneamente a estos cambios sociales, España experimentó un fuerte crecimiento poblacional debido a los procesos migratorios, que la convirtieron en uno de los principales destinos para las personas provenientes de América Latina y el Caribe. Este crecimiento se manifestó en un aumento considerable de los extranjeros, cuando se pasó de 923879 en el año 2000 a 5747734 comenzando el 2010, según datos del Instituto Nacional de Estadística Español (INE), es decir, que en una década se sextuplicó el número de inmigrantes.

El reconocimiento de los derechos de la comunidad LGBTI se ha convertido en un factor de atracción para aquellas personas que sufren algún tipo de discriminación, ya sea institucional, legal, social o cultural, por su condición sexual en sus países de origen; sin embargo, no son solo quienes se ven privados de sus derechos los que migran a España: también lo hacen hombres y mujeres que buscan espacios sociales más tolerantes y respetuosos que les garanticen el disfrute pleno de sus derechos.

Es así, como los estudios sobre migración han dado cuenta que "Ios migrantes son mucho más diversos en términos de identidad sexual y de género de lo que se ha considerado, ya que no todos los hombres y mujeres que migran son heterosexuales, y es necesario entonces estudiar una nueva realidad de la migración donde la identidad sexualidad y de género permita romper las visiones reduccionistas que niegan su existencia en este ámbito" (Restrepo, 2013, p. 36).

Publicaciones más recientes como la de Restrepo (2016) pone de manifiesto como el proceso migratorio influye de manera directa en las percepciones que tienen los sujetos que migran en aspectos tales como la salud. Por su parte, Dhoest (2016) explora el papel que cumplen los medios de comunicación de masas y en línea en la formación de la identidad sexual de los hombres gais de origen inmigrante que viven en Bélgica. Por su parte, Wimark (2016) sostiene que los patrones de migración de los hombres homosexuales son cada vez más similares a los de la población general para el caso de los migrantes que han Ilegado a Suecia.

El presente artículo, tiene como objetivo el estudio de las experiencias personales y las trayectorias sociales de los varones homosexuales y bisexuales colombianos que han migrado a España. Analizando sus experiencias desde la consideración de las percepciones, prácticas y comportamientos que tienen que ver con la construcción de la identidad sexual y cómo influye esta en las motivaciones y la decisión de migrar. En algunos casos la identidad sexual aparece como un factor determinante 
para dar inicio al proceso migratorio y en otros se relaciona con aspectos personales y sociales que pueden consolidar dicho proceso. Algunos de los varones emigrados establecen relaciones de pareja en el país destino o perciben en este una sociedad más respetuosa que brinda mayores garantías para el desarrollo de una vida plena. En otras ocasiones, es la familia quien conocedora de la orientación sexual de sus hijos opta por brindarles la posibilidad de llegar a un país donde no estarán sometidos a actos de discriminación.

Es así como los varones homosexuales y bisexuales entrevistados establecen una serie de estrategias migratorias que se valen de sus redes sociales y de sus expectativas personales para consolidar sus procesos migratorios.

\section{METODOLOGÍA}

Las experiencias de los varones homosexuales y bisexuales migrantes no pueden entenderse de forma aislada. Su análisis debe partir desde una perspectiva relacional, donde los países de origen y de destino permitan entender las relaciones de género y la sexualidad de estos varones.

Se hace necesario, entonces, conocer cómo los varones homosexuales y bisexuales de origen colombiano construyen su sexualidad en función de los valores, las normas y la cultura de su país de origen, y cómo la experiencia migratoria en España repercute en la reconfiguración de sus vidas afectivo sexuales y de sus concepciones de género que modifican su identidad.

Este análisis debe estar enmarcado en el contexto social de destino, puesto que los migrantes han de adaptarse simultáneamente a los conceptos sociales dominantes de género y sexuales presentes en este y, además, a las identidades sociales marginales, tales como ser colombianos e inmigrantes.

Para aproximarnos a esta realidad la investigación ha utilizado tanto el enfoque cuantitativo como cualitativo, que permitió complementar entre sí la información recopilada por ambos medios, considerando en cada caso el tipo de información que se requiere.
El trabajo de campo se desarrolló a través de entrevistas en profundidad, que se establecieron desde el inicio de la investigación como la herramienta metodológica fundamental con el fin de proceder a la consecuente elaboración de los discursos de los entrevistados que integrasen la dimensión emic. Según Harris (1979), la perspectiva emic proviene del informante nativo, es decir, el observador emplea e intenta esclarecer categorías que el nativo considera reales y que otorgan sentido a su actuación.

Además, «el investigador no es un mero recopilador, un mero coleccionista, sino que proporciona información elaborada» (Velasco y Díaz de Rada, 2003 , p. 35), pues a través del proceso de producción de información interviene e interactúa de forma constante con los sujetos objeto de la investigación, permitiendo mostrar una descripción de experiencias vividas en primera persona y los contextos en los cuales esas mismas experiencias se han ido concibiendo y redefiniendo (Bertaux, 2005).

La observación participante adquiere, a 10 largo de todo el proceso de trabajo de campo, un papel fundamental que va más allá de la función de complementariedad, pues esta se convierte en una herramienta que articula espacios, tiempos y discursos.

Así, se da origen a la perspectiva etic, que se vincula con la capacidad del observador para generar categorías y conceptos desde un punto de vista científico, para proveer de significado las situaciones sociales y culturales que se analizan.

Por otra parte, fue necesario tener en cuenta que las investigaciones que abordan el tema de la sexualidad requieren de ciertas adecuaciones metodológicas, pues "las preguntas de carácter sexual resultan ser muy intrusivas de la intimidad y, por lo tanto, no siempre son contestadas por las personas de forma fiable, y en algunas ocasiones suelen ser proclives a responderse de la forma más aceptada socialmente" (Baile, 2008 , p. 153). Por tanto, el sistema de referenciación permitió tener un mayor grado de confianza entre el entrevistador y el entrevistado ya que de antemano este último conocía la experiencia previa de quien le había invitado a participar en la investigación. 


\section{Población objetivo}

Según los datos del INE (2011), la población migrante colombiana residente en España es de 273176 personas, de las que 121748 son varones y 151428 mujeres. Los inmigrantes colombianos se concentran en las Comunidades Autónomas de Cataluña, Valencia y Madrid, con una cifra en conjunto de 150044 personas; le siguen Andalucía y Canarias, con 21629 y 18 864, respectivamente.

El 83,6\% de los varones inmigrantes colombianos forman parte de la población en edad económicamente activa, es decir, de la población que tiene la capacidad de incorporarse al mercado laboral. El $15,4 \%$ está representado por niños y adolescentes hasta los 16 años, y solo el 1,1 \% corresponde a hombres de 65 años y más.

Esto quiere decir que la población de varones inmigrantes colombianos residentes en España está conformada en su mayoría por jóvenes y adultos. Los primeros incluyen a los adolescentes y jóvenes en sus últimas etapas hasta la llegada a la vida adulta hacia los 22-25 años, siendo un grupo significativo en cuanto a los procesos demográficos que suceden en este periodo, entre ellos, la edad de entrada para la vida en pareja, la edad de la paternidad, la finalización de la preparación educativa y profesional y el acceso al mercado de trabajo.

Siendo los colombianos uno de los mayores colectivos de inmigrantes en España y reconociendo que este grupo de población es una de las más dinámicas y activas en el ámbito social y económico, que además fortalece muchos de los procesos sociales actuales, entre ellos, las migraciones internacionales, se decide abordar dicho colectivo como población objetivo.

\section{Diseño y selección de la muestra}

En cuanto al número de entrevistas realizadas, estas no responden a una estricta cuestión de diseño y planificación, sino al hecho de considerar la saturación de la información recopilada, buscándose fundamentalmente la profundidad y privilegiándose la calidad de la muestra sobre la cantidad.

Por otra parte, el tamaño de la muestra no se define a priori, éste se perfila de acuerdo a las unidades de análisis pero la muestra definitiva se determina cuando al agregar más casos éstos no aportan información o nuevos datos, de tal modo que se alcanza la saturación conceptual de categorías.

Para el diseño y la selección de la muestra se utilizó el muestreo en bola de nieve, el cual tiene como idea central el hecho de que cada individuo de una determinada población pueda referenciar otros individuos en ésta, los cuales tienen la misma probabilidad de ser seleccionados. Así, a cada individuo que es escogido, se le pide que referencie a otros individuos, siendo una condición fundamental el hecho de que el primer grupo de entrevistados es seleccionado de manera aleatoria.

\section{EXPERIENCIAS MIGRATORIAS}

En total se realizaron 34 entrevistas en España; la mayor parte de estas en las Comunidades Autónomas de Madrid y Valencia, y en menor proporción en otras Comunidades como Cataluña y Andalucía. Los datos socioeconómicos de los entrevistados hicieron evidente el hecho de que la movilidad es mucho mayor entre los adultos jóvenes, pues 21 de los varones interrogados tenían edades comprendidas entre los 18 y los 27 años. La edad promedio de los migrantes entrevistados es de 27,6 años, quienes en la mayoría de los casos han migrado antes de cumplir los 23 años de edad, lo que corresponde con la finalización de los estudios medios, técnicos 0 superiores y el inicio de la vida laboral.

Por edades, los varones entre los 18 y los 32 presentan tiempos de residencia en España que se concentran entre 2 y 3 años, es decir, periodos relativamente cortos comparados con los de los hombres mayores de 38 años que han residido 6 años 0 más.

Esto se puede explicar en el sentido de que los hombres que han migrado recientemente lo han hecho por motivos académicos o laborales, los cuales llegaron mayoritariamente a través de contingentes de trabajadores no comunitarios. En ambos casos estos no superaban los 27 años de edad.

En cuanto al nivel educativo se observa que 3 hombres entrevistados tienen completa la educación secundaria obligatoria, 17 han completado 
el bachillerato, 1 posee estudios tecnológicos, 10 varones tienen estudios universitarios y 3 entrevistados cuentan con estudios de posgrado.

Por tanto, casi la mitad de los entrevistados (14 migrantes) tienen una alta cualificación académica, es decir con estudios tecnológicos o superiores que les permitiría incorporarse al mercado laboral de manera más efectiva. Sin embargo, esto no sucede así, ya que se desempeñan como becarios 0 cumpliendo actividades que no corresponden a su nivel formativo.

Por otra parte, el análisis de la migración según el estado civil de las personas permite determinar la relación entre los procesos migratorios y las etapas del ciclo vital, debido a que "existe la expectativa social de que las personas al casarse establezcan un nuevo hogar (si bien la migración hacia ese nuevo hogar puede muy bien producirse ahora antes del matrimonio formal)" (Weeks, 1990, p. 196).

Pero esto no tiene una relación directa con las parejas del mismo sexo, ya que, como se observa, la mayor parte de los entrevistados son solteros (34), solo 2 se encuentran casados e igual número corresponde a quienes tienen registrada una pareja de hecho.

Aunque estos hombres manifiesten estar solteros no significa que no tengan una pareja estable, solo hace referencia a que no cuentan con un vínculo legal para formalizar dicha convivencia. En consecuencia, es necesario profundizar en la dinámica de este tipo de relaciones, ya que sus implicaciones para la sociedad de acogida y la integración de los inmigrantes varían mucho en ámbitos tales como la vivienda, la educación o la salud.

Respecto a la situación laboral, 14 de los entrevistados son empleados, 9 se encuentran desarroIlando labores académicas o como becarios (a este número corresponden aquellos hombres que tienen una estancia por estudio o investigaciones en España). Le siguen 7 varones que se encuentran en situación de desempleo, sólo 1 es autónomo y 3 se dedican al trabajo sexual. Es necesario aclarar que aquellos varones que se dedican al trabajo sexual no llegaron a España para ejercer dicha actividad, sino que las condiciones laborales y sociales los han empujado a esta. Así lo manifiesta uno de los entrevistados:
"Pues la verdad me fui de chapero ${ }^{1}$ a una discoteca, usted sabe que chapero es prostituto, porque usted sabe que la situación es difícil y entonces me fui a buscar una ayuda allá" ES_Harold_26

Durante la realización de las entrevistas se constató que existen factores personales y sociales que pueden condicionar que los migrantes se dediquen al trabajo sexual. Este es el caso de algunos de los entrevistados que, aun contando con permiso de trabajo, confiesan haberse dedicado a la prostitución masculina por otros motivos diferentes al desempleo o la necesidad económica.

"Me ofrecieron dinero una vez, estando sentado en la barra en una discoteca que yo no sabía que era para chaperos. Que ni idea, yo no sabía qué era un chapero. Un señor me ofreció, me preguntó que si era chapero y yo no le entendí." ES_Camilo_19

Este discurso pone de manifiesto que algunos de los estereotipos que se manejan frente a los inmigrantes latinos y jóvenes en España los conducen hacia nuevas experiencias que no necesariamente son buscadas por los migrantes, incluyendo el trabajo sexual. Es así, como algunos de los entrevistados se han visto abocados a la prostitución tras experiencias personales en sitios de ocio y diversión, donde estos hombres desconocían que se ejercía el trabajo sexual masculino y las normas que rigen dicha actividad dentro de este tipo de lugares.

Las experiencias migratorias nos permiten identificar las condiciones y las estrategias que emplean los migrantes para llegar a su destino. En el caso de España, se hace necesario entonces indagar la experiencia migratoria de los entrevistados y las redes sociales que les han permitido desarrollar el proyecto migratorio, así como las redes de acogida. Todos estos aspectos permiten caracterizar los movimientos migratorios desde diversos aspectos, que luego darán cuenta del tipo de migración empleado por cada uno de los migrantes.

1 Real Academia Española. Chapero: homosexual masculino que ejerce la prostitución. 
De manera general, los entrevistados proceden de la Región Andina y del departamento del Valle del Cauca, lo cual corresponde con los tres departamentos con mayores tasas de emigración ${ }^{2}$ en $\mathrm{C}_{0}$ Iombia son Risaralda, Valle del Cauca y Quindío con el $29,84 \%, 19,66 \%$ y $18,90 \%$ respectivamente (Khoudour-Castéras, 2007, p. 260).

El municipio de Dosquebradas en el departamento de Risaralda, según el censo de 2005, se ubica como una de las primeras ciudades con mayor porcentaje de hogares con experiencia migratoria, llegando al 11,6 \%, y son España (55,8\%) y Estados Unidos (29,6\%) los principales destinos migratorios para las personas de este municipio.

Por su parte Pereira, como capital del departamento de Risaralda, presenta un porcentaje del $10,2 \%$ de hogares con experiencia migratoria, con una participación también mayoritaria de la emigración hacia España.

\section{Experiencia migratoria}

En algunos casos, los entrevistados manifestaron haber tenido experiencias migratorias a países como Estados Unidos o Venezuela; sin embargo, dichos procesos migratorios no tuvieron el éxito que ellos se habían esperado, y han optado por regresar a Colombia y emprender un nuevo proyecto migratorio hacia España.

"Salí de estudiar bachillerato me fui para Miami, en Miami no me funcionaron las cosas porque igual yo solo tenía permiso de, iba como turista y hasta estudiar era ilegal porque iba de turista" (ES_Camilo_19)

En otros casos, España se convirtió en la segunda opción como destino migratorio, debido a que después de los atentados del 11 de septiembre de 2001 en Estados Unidos los controles migratorios aumentaron, cosa que dificultó la entrada de migrantes en este país.

2 El número de emigrantes por departamento se calcula a partir del número total de emigrantes colombianos y la distribución de los hogares con emigrantes. La tasa de emigración es la relación entre el número de emigrantes y la población.
"En principio íbamos a ir a los EE. UU. pero en esa misma época pasó lo de las Torres Gemelas y todo cambió de rumbo, ya que España era uno de los que no tenían en ese momento visa, optamos por eso" (ES_ Samuel_28)

Por otra parte, algunos autores sostienen que "muchos hombres pertenecientes a las minorías sexuales buscan emigrar a los Estados Unidos y a sus territorios. Esto plantea enormes dificultades vinculadas al idioma, cultura, mercado de empleo y capacidad del emigrante para insertarse de forma efectiva a la nueva sociedad" (Toro. J. López, M. y Nieves, K, 2012, p. 67).

Los flujos migratorios de colombianos hacia Estados Unidos y Venezuela se remontan a las décadas de los sesenta y setenta, lo que generó fuertes redes sociales que permitían la movilidad de personas entre estos países y consolidaron a los Estados Unidos como destino migratorio por excelencia. Las condiciones económicas que ofrecía este país a los inmigrantes a mediados de la década de los setenta permitió la diversificación de los migrantes, dando "cabida ya no solo a profesionales universitarios sino también a obreros no calificados, comerciantes y empresarios de clase media" (Ramírez et al., 2010:18). En la década de los noventa, sin embargo, las políticas migratorias de los Estados Unidos y Venezuela se fortalecieron, aumentando las restricciones legales a la inmigración hacia estos países, lo que originó una diversificación en cuanto a destinos y características de los migrantes. Aparece entonces España como uno de los principales destinos, aprovechando que los colombianos no requerían visa para viajar a este país, la visa fue implantada a partir del año 2001.

Podría afirmarse que la experiencia migratoria de los entrevistados es muy poca y que el éxito de los proyectos migratorios se basa en la utilización de las redes sociales ya consolidadas, para tal fin los migrantes han recurrido a sus familias 0 amigos para emprender el proceso. Por otra parte, en el caso de aquellos que han tenido mayores experiencias migratorias, estas se encuentran asociadas con el nivel económico del migrante y a expectativas de tipo social y cultural, como mejorar su nivel académico 0 conocer nuevas culturas. 


\section{Redes sociales}

Las redes sociales se convierten en elementos fundamentales que facilitan el proceso de entrada en España y el proceso de acogida en el país, de modo que dichas redes establecen el éxito de los proyectos migratorios, ya sean individuales 0 familiares. Así, "estas redes proporcionan cuestiones esenciales, como son el primer alojamiento, los primeros contactos para encontrar un empleo y la información necesaria para iniciar los trámites exigidos hasta que se consiguen los primeros papeles" (INE, 2007).

Todos los migrantes entrevistados contaban con una red de apoyo para iniciar el proceso migratorio y una red de acogida al llegar a España. Estas dos redes no siempre eran las mismas: la primera ofrecía al inmigrante la información necesaria para salir del país (y en algunos casos, la ayuda económica para emprender el viaje), mientras que la red de acogida, por su parte, les aseguraba un alojamiento, la alimentación y una guía para movilizarse dentro de la ciudad y encontrar un trabajo. Para Lomnitz (1981), las redes sociales son una forma de brindar apoyo, ya sea moral, social o económico con relación a determinadas condiciones como la vecindad física que facilita el flujo continuo de intercambio. Además, estas redes reflejan la disposición de establecer y consolidar una relación e intercambio recíproco entre los implicados. Por tanto, la red es de carácter dinámico a la cual se pueden sumar nuevos miembros.

"Un amigo, un amigo gay fue el que me ayudó, yo venía para donde mi prima, porque me daba el alojamiento, pero para venirme me ayudó un amigo que estudió conmigo en el colegio, él ya llevaba tiempo aquí, él fue el que me prestó los dólares para pasar, la carta de invitación, que aunque luego eso aquí me toco pagárselo, pero él fue el que me ayudo"(ES_Alberto_40)

Se hace evidente en este caso la diferenciación entre la red de apoyo para iniciar el proceso migratorio y la red de acogida, las cuales se complementan y contribuyen al éxito del proyecto migratorio. Así, los familiares brindan el apoyo económico y social para iniciar la migración, pero los amigos pro- porcionan el apoyo moral y social para instalarse en el país de destino, determinado por la identidad sexual que comparten los involucrados.

Esta situación se evidenció en los discursos de algunos de los entrevistados, ya que debido a la identidad sexual del inmigrante estos prefieren la independencia y el anonimato para desarrollar su sexualidad, alejado de los controles de tipo familiar.

"Vine a vivir a la casa de un amigo, de un amigo que tenía en Colombia que vino seis meses antes que yo" (ES_Edwin_28)

En cuanto a la migración familiar, y específicamente a las reagrupaciones familiares, las redes de apoyo para emprender el viaje como las redes de acogida estaban constituidas por los miembros de la familia, ya sean padres y/o madres, aunque en otras circunstancias estas las integraron primos u otro tipo de parientes cercanos.

"Directamente de mi familia, mi madre, llegué a vivir con mi madre y con mi hermano, en aquel entonces vivían ellos solos y llegué a vivir yo con ellos, entonces económicamente todo dependía de mi madre" (ES_Nelson_23)

Otro tipo de apoyo para dar inicio a los procesos de reagrupación familiar provenía de algunas organizaciones no gubernamentales en temas referidos a los requisitos y trámites necesarios, así como asesoría jurídica en dichas cuestiones.

En síntesis, puede decirse que la intensidad de los procesos migratorios entre Colombia y España en los últimos años ha llevado a la consolidación de las redes migratorias que han permitido el aumento de su densidad, la cual es directamente proporcional a la intensidad del proceso migratorio. Esto ha permitido además que las trayectorias migratorias están bien definidas, determinando, entre otras cuestiones, las estrategias de reagrupación familiar y los nuevos procesos migratorios.

\section{Motivaciones y expectativas del proceso migratorio}

Las motivaciones del proceso migratorio pueden ser muy variadas, sin embargo, algunos autores 
como Berry han definido dos factores fundamentales: "los factores que repelen ("push"), el deseo de escapar de situaciones poco placenteras que se dan en el país de origen (problemas personales, económicos, políticos, familiares). Los factores que atraen ("pull"), lo atractivo de vivir en el extranjero (prestigio de un grado educativo en el extranjero, oportunidad para el crecimiento personal, deseo de una nueva experiencia)" (Berry et al., 1987 citado en Farjado et al., 2008, p. 45).

El elemento determinante de los procesos migratorios de estos hombres entrevistados es su identidad sexual, que se relaciona con los factores que repelen y aquellos que atraen definidos por Berry. Es así como existen una serie de relaciones entre las diferentes motivaciones para dar inicio al proceso migratorio, en algunos casos vinculados con su identidad sexual, mientras que en otros juega un papel como mediador dentro del proceso, hasta encontrar aquellos donde deja de ser un factor definitivo para tal fin.

Es así como "las expectativas económicas y sociales pueden generar un proceso migratorio laboral 0 las motivaciones familiares pueden desembocar en un proceso de reagrupación familiar. Sin embargo, la migración sexual es un asunto más complejo, ya que de ella forman parte una serie de elementos de tipo social, económico o familiar que, sumados, darían como resultado este tipo de migración" (Restrepo, 2015, p. 435).

Este apartado dará cuenta de las diferentes razones y motivaciones por las cuales los varones homosexuales y bisexuales entrevistados han decidido emprender su proceso migratorio a España y las experiencias que se han desprendido de este hecho. Además, de establecer una serie de tipologías migratorias que se desprenden de los discursos de los entrevistados.

\section{Migración laboral}

Según la Organización Internacional de las Migraciones (0IM), la migración laboral se define como el movimiento de personas desde el país de origen a otro con objeto de trabajar, cuya finalidad es mejorar el nivel de vida propio y el de sus familias.
Entre los varones colombianos que llegaron por motivos laborales se encuentran aquellos que contaban con empleos precarios en su país de origen y querían aumentar sus ingresos. Al llegar a España, sin embargo, la precariedad aumenta, ya que sus empleos no corresponden con su formación profesional, deben desempeñar amplias jornadas laborales y, en algunos casos, no cuentan con contrato laboral. A pesar de esto, los ingresos sí presentan un aumento considerable, lo que de alguna forma satisface en parte dichas expectativas.

A este grupo se suman aquellos que aun contando en su país de origen con una situación profesional y económica buena, han decidido migrar por razones sociales, para mejorar sus capacidades académicas y así aumentar su reconocimiento profesional; sin embargo, lo que se evidencia en las entrevistas es que estos varones no regresan a su país de origen después de terminar su formación y permanecen en España.

"Cuando decidí venirme a España quería venir a estudiar. Sin embargo, eso era una excusa pues mi idea era quedarme aquí y trabajar, sin olvidar los estudios que también me interesaban, pero quería trabajar" (ES_Cesar_28)

Este testimonio también corrobora lo planteado por Orelllana "las migraciones que comienzan con la excusa educativa nunca son solo eso, el aprendizaje que se busca no es así ingenuo, ni el motivo real de la movilidad está atravesado por las universidades" (Orellana, 2014, p. 45). Es decir, la educación se convierte en una "excusa" para adquirir otro tipo de experiencias personales que pueden estar vinculadas con la identidad de género o la orientación sexual de quien migra.

Por otra parte, se encuentran aquellos hombres que llegaron a través de contingentes de trabajadores extranjeros no comunitarios para desempeñar ocupaciones especialmente en el sector de la salud y los servicios.

"En un convenio que tiene el Sena (Servicio Nacional de Aprendizaje de Colombia) con España para traer a trabajar empleados de salud de enfermería [...] para venir a trabajar a residencias geriátricas." (ES_Edwin_28) 
Dentro de este tipo de migración se encuentran 21 de los varones entrevistados. Como ya se ha mencionado, se incluyen en este grupo los migrantes que llegaron a través de los contingentes de trabajadores no comunitarios, aquellos que emprendieron el proceso migratorio por motivaciones académicas pero que finalmente se quedaron en España trabajando y quienes manifestaron otro tipo de motivaciones sociales pero que actualmente residen en este país.

\section{Migración familiar}

Dentro de la migración familiar se han considerado dos formas diferentes. La primera de ellas se refiere a los procesos de reagrupación familiar, es decir, al derecho de los extranjeros residentes en España a la vida en familia. Para ello pueden reagrupar a sus familiares donde son reagrupables según las leyes migratorias españolas, el cónyuge y los hijos menores de edad de ambos, así como la pareja de hecho y el ascendiente de primer grado del reagrupante, siempre y cuando se cumplan los requisitos establecidos en la normativa aplicable.

A este tipo de migración corresponden 7 de los varones entrevistados, los cuales Ilegaron a España a través de un proceso de reagrupación familiar; siendo los varones más jóvenes, que no tuvieron ningún tipo de decisión dentro de la elección del destino migratorio, ya que al ser menores de edad dicha elección la realizaron sus padres.

"Bueno porque mi madre vino mucho antes, se vino a trabajar y cuando yo tenía once se presentó la oportunidad de que viniéramos mis hermanos y yo y pues vinimos, básicamente por eso" (ES_AndrésFelipe_22)

Dentro de los procesos de reagrupación familiar, destacan aquellos que fueron motivados por razones de identidad sexual de los hijos de los inmigrantes que se encontraban aún en el país de origen. Es decir, los hijos homosexuales o bisexuales de padres que estando en España tomaron la decisión de traerlos desde Colombia, país donde residían, con la finalidad de que pudieran vivir en un ambiente más respetuoso y libre. Dicho proceso se considera como migración sexual y será analizado en su respectivo apartado.
En segundo lugar, se encuentra la migración referida a la vida en pareja, cuyas expectativas tenían relación directa con la formación de una familia y los derechos vinculados a esta, derechos que no se encuentran asegurados en su país de origen. En este caso se hablaría de una migración sexual. Sin embargo, existen parejas que han iniciado el proceso migratorio en busca de nuevas oportunidades laborales 0 en un cambio en su contexto social.

"Viajé con mi pareja desde Colombia motivados por las oportunidades que habían en España con respecto a los ingresos económicos" (ES_Lucas_28)

Se consideran estrategias migratorias familiares aquellas que involucran a la pareja como parte primordial en el proyecto migratorio con el fin de conseguir una estabilidad económica 0 social deseada, sin que esto implique necesariamente la búsqueda de garantías sociales frente a derechos relacionados con la familia.

\section{Migración sexual}

La migración sexual está "motivada totalmente 0 en parte, por las sexualidades de aquellos que migran, ello implica una combinación de motivaciones económicas y sexuales" (Carrillo et al., 2008 , p. 7). Para ser más precisos, se podría decir que es un proceso donde la identidad sexual ${ }^{3}$ del migrante cumple un papel fundamental para motivar su desplazamiento a otro contexto; donde los aspectos sociales, económicos y políticos, tanto del país de origen como el de destino, determinan las experiencias migratorias de estas personas. Tal es el caso de los varones entrevistados que han tenido experiencias migratorias anteriores a otros países 0 aquellos que han migrado para estudiar y luego se establecen definitivamente en el país de destino.

\footnotetext{
3 La identidad sexual entendida como el autoconcepto que tiene cada persona y en el cual se relacionan el sexo, el género, la orientación sexual, la identidad de género y el conjunto de habilidades con las que la persona se desenvuelve en su vida y en relación a la sexualidad.
} 
Se puede decir que la migración de tipo sexual surge como resultado de ciertos controles sociales, entendidos estos como los mecanismos que desarrolla la sociedad a través de diferentes agentes y mecanismos que garantizan la aceptación por parte de sus miembros, de sus normas, valores, intereses y pautas de conducta de manera voluntaria 0 forzada. Cuando estas pautas son impuestas y van en contra de la identidad propia de las personas, limitan su crecimiento personal. Sin embargo, "el control social dependerá del grado en el que el individuo asuma las actitudes de aquellos que están implicados con él en sus actividades sociales" (Mead, 1991, p. 184) en los ámbitos de la familia, la religión y el círculo social más próximo, quienes definen cómo y de qué manera se debe desarrollar la vida de un hombre en un contexto de heteronormatividad. Además, confluyen aspectos sociales, culturales y económicos que en definitiva darán origen a un proceso migratorio en el que el factor concluyente es la identidad sexual.

El análisis de la migración sexual debe considerar todos los aspectos sociales, culturales, políticos y económicos vinculados con el migrante, pues en algunos casos la relación entre la identidad sexual y la migración no es evidente, debido a que puede estar justificada bajo otro tipo de motivaciones que, sin embargo, son parte integral de un proceso migratorio estructurado en torno a la identidad sexual de quien migra.

En este sentido, algunos de los inmigrantes manifiestan durante las entrevistas que sus motivaciones para iniciar el proceso eran de tipo social (por ejemplo, continuar 0 iniciar estudios de posgrado). Sin embargo, al avanzar en las entrevistas se podía constatar que sus parejas se encontraban residiendo en España incluso antes de que ellos llegaran, lo que claramente constituiría una motivación vinculada a su relación afectivo-sexual y, por ende, a su identidad sexual, sin que esto le reste valor a las motivaciones de tipo social o económico, pues estas se complementan.

Esta misma situación se evidenció en otra investigación al concluir que "aunque este grupo (hombre dominicanos gay) aseguraba entre sus prioridades, tener esperanzas de un progreso académico y económico, estaba entre sus relatos un futuro de una vida sexual sin represiones que atentara con la salud emocional de estos, lo que define el concepto de migración sexual" (Toro, J., López, M. y Nieves, H. 2012, p. 73).

A este tipo de migración corresponden 6 de los entrevistados, los cuales ponen de manifiesto que esta surge como respuesta a una serie de controles sociales en Colombia que limitan el pleno desarroIlo de la persona. Dichos controles son de tipo informal, y puede ejercerse de diversas maneras: "el ridículo, la risa, o mediante el uso amenazante de cualquiera de estas técnicas. La mera ocasión de perder la aceptación del grupo usualmente es suficiente para hacer que la persona actúe de acuerdo con las expectativas" (Cohen, 1999, p. 99).

En el análisis de los discursos de los entrevistados se evidenciaron las siguientes estrategias para superar el estigma y la discriminación en su país de origen:

\section{Estrategia de localización en busca de aceptación social}

La estrategia de aceptación social hace referencia a los procesos migratorios en los que se evidencia el papel de la identidad sexual dentro de este; en otras palabras, es la necesidad personal del migrante para hacer frente a sus requerimientos afectivos y sexuales de forma libre, segura y satisfactoria, permitiéndole superar la imposibilidad o la dificultad de asumir la identidad propia en su país de origen.

"El bullying ${ }^{4}$ que había vivido en el colegio, que había vivido en ciertos espacios, pues que la pasé fatal, yo decidí por ejemplo perder un año, perdí décimo de bachillerato con el objetivo de no seguir con los truhanes que estaba, porque me hicieron la vida imposible de noveno a décimo" (ES_Victor_33)

4 Bullying es el término en inglés para el acoso escolar. También conocido como "hostigamiento escolar" o "matoneo escolar", es cualquier forma de maltrato psicológico, verbal o físico producido entre escolares de forma reiterada a lo largo de un tiempo determinado. 
Este tipo de situaciones presentes en Colombia tienen su origen en una cultura machista, en la que se discrimina y ridiculiza a aquellos varones que puedan tener ciertos rasgos considerados femeninos o que expresan su afecto a una pareja de su mismo sexo, actos considerados reprochables dentro de la "normalidad" afectivo-sexual vigente en este país.

La estrategia de aceptación social, al igual que otras abordadas más adelante, buscan romper los controles sociales que se ejercen en el país de origen, aquellos que tienen que ver con los medios informales, es decir, las normas morales, las costumbres, las reglas de trato social y la moda, entre otros. Todos ellos tienen una base común: la heteronormatividad, gracias a la cual son reproducidos y transmitidos en forma de hábitos, normas y valores determinados.

Es así como la familia, la iglesia y el círculo social más próximo (el barrio y los amigos) constituyen elementos de los que se vale la sociedad y la clase dominante para ejercer e imponer sobre el resto normas de conducta, valores morales y éticos y su ideología, de acuerdo a las funciones que realicen cada uno de ellos.

Por otra parte, la estrategia de aceptación social busca superar la homofobia que se vive en el contexto social de origen, homofobia entendida como "el prejuicio, estigma 0 discriminación hacia las personas que mantienen relaciones sexuales con otras de su mismo sexo" (OPS, 2006, p. 7).

"En Colombia hasta muchos chicos jóvenes si ven una pareja de gais pues empiezan a chiflarle 0 a gritarle por cómo vayan vestidos, si van muy afeminados, entonces yo sé, eso lo viví yo, se veía en Colombia, de que los mismos chicos les gritaban cosas"(ES_Alberto_40)

Se ha constatado, a través de las entrevistas, la homofobia que viven los varones con diversas orientaciones sexuales en su país de origen, la cual es aceptada, tolerada y considerada normal por estos; es decir, las ridiculizaciones e insultos son entendidos como parte de un tipo de prácticas sociales vinculadas con la identidad sexual.

\begin{abstract}
"Así como hay algunas personas que no les gustan los perros o hay algunas personas que no le gustan las gordas o ven mal un negro, hay personas que nos ven mal a nosotros y completamente normal" (ES_Sebastian_20)
\end{abstract}

El hecho de carecer de ciertos derechos no representa para algunos hombres un inconveniente, situaciones que han permitido perpetuar la discriminación hacia las minorías sexuales, basadas en una autoaceptación como "anormales" dentro de un contexto heteronormativo.

"En cuanto a las oportunidades es igual tanto para los gais como para cualquier tipo de personas, lo único es que no se pueden formar hogares ni nada de esto, pero del resto es igual como cualquier otra persona" (ES_Fidel_25)

Por lo tanto, la homofobia se ha interiorizado y constituye parte del ser homosexual o bisexual en el contexto social colombiano, situación que se confronta al llegar a España, donde se toma conciencia de que dichos comportamientos sociales son degradantes, estigmatizantes y discriminadores.

"De una escala de 1 a 10, para mí era normal aguantarme los insultos de 1 a 6 , era normal que me insultaran, yo no lo veía como un insulto, aquí me enteré que eran insultos y eso no solo me pasa a mí, eso le pasa a otras personas colombianas de diferentes ciudades"(ES_Victor_33)

Sin embargo, estos varones en España no están libres de tales situaciones, y se pueden llegar a enfrentar a una doble discriminación: por una parte, la que surge de la homofobia, y por otra, la que genera su condición como inmigrantes.

\section{Estrategia de reagrupación familiar}

Se considera como estrategia de reagrupación familiar la que tiene relación directa con la identidad sexual del reagrupado, en este caso los hijos de los migrantes que se encontraban en el país de origen. Dicha estrategia puede surgir por deseo propio 
del hijo o como respuesta de la familia a situaciones que perjudican el desarrollo personal de este en un contexto social como el colombiano.

"En sí yo no quería venir, si no que como mi madre ya está acá hace cinco años nos reagrupó $[\ldots]$ cuando vivía en Colombia dije: ya me cansé de decir tantas mentiras, tantas mentiras, le voy a contar a mi madre que soy homosexual y se lo dije y nada, ella decidió traerme para acá, porque acá la vida era más fácil" (ES_Sergio_19)

La decisión tomada por parte de los padres sobre el hecho de reagrupar a un hijo por razones de su identidad sexual se basa en su propia experiencia personal en el país de destino, la cual se compara con aquellos contextos sexuales del país de origen y con las experiencias vividas allí.

Dicho proceso les ha permitido tomar conciencia sobre la diversidad sexual y asumirlo de una forma normalizada, gracias además a que en España el tema es abordado en todos los ámbitos sociales y culturales de manera constante y libre, desvirtuando las ideas y los imaginarios con los que podrían haber llegado estas personas.

"En realidad no fue porque mi madre estaba aquí, fue por un tema personal, personal de [...] mi familia colombiana sabía que era homosexual, era un conflicto y mi madre me ha dicho vente a España que nadie te va a decir nada y vas a vivir perfectamente y por eso, he venido, básicamente" (ES_Luis Carlos_26)

La sensibilización frente a la diversidad sexual y la identidad de género ha permitido influir en las percepciones de los inmigrantes, logrando aceptar una realidad que en el país de origen estaría condicionada por elementos sociales arraigados en una cultura tradicional con fuertes elementos de machismo. Las ideas, las prácticas y las percepciones de los inmigrantes fluyen entre el país de destino y el de origen, pudiéndose considerar como remesas sociales que intervienen en las relaciones de las familias y la identidad cultural, y permiten dar origen a cambios sociales en Colombia.
Se puede decir, entonces, que los padres han optado por reagrupar a sus hijos porque consideran que en España existen las garantías legales y sociales suficientes para que estos desarrollen una vida plena acorde a su identidad sexual.

\section{Estrategia de vida en pareja}

A este tipo de estrategia responden aquellos procesos migratorios que conllevan a consolidar una pareja. Se presenta cuando uno de los hombres se encuentra en el país de destino 0 en algunos casos cuando ambas personas inician el proyecto migratorio.

"Estaba enamorado, por eso llegué acá España, y mi pareja vivía acá y él me trajo" (ES_Fabricio_21)

"Cuando llegué aquí ya vi que hay más relaciones estables, una pareja homosexual es lo mismo que una pareja heterosexual, se pueden casar, tener hijos y formar una familia, ese concepto no lo veía en Colombia"(ES_Sergio_19)

La finalidad de la estrategia es lograr superar los controles sociales impuestos por la familia 0 el círculo social próximo y la consolidación de una relación de pareja en el destino donde se pueda vivir en familia y tener acceso a todas las garantías legales con las cuales cuentan las parejas heterosexuales. De manera adicional, se busca superar una idea muy arraigada en el imaginario colectivo colombiano, en el que los homosexuales no pueden formar una familia y están condenados a la promiscuidad y a vivir en soledad.

Por otra parte, aunque menos frecuente, se encuentran aquellos casos donde uno de los hombres viajó desde Colombia para conocer a la persona con la que había entablado una relación afectiva a través de internet. Dichas relaciones se construyeron a través de conversaciones en el chat, videoconferencias 0 llamadas telefónicas, donde ambos varones pudieron compartir sus expectativas respecto a la relación que deseaban entablar. Además, tuvieron la oportunidad de conocerse físicamente a través de fotografías y videoconferencias, lo que permitió tomar la decisión 
de migrar contando con el apoyo de la persona en España.

"A mí me trajo un novio, él me quería hacer el coprológico (Scat $\left.{ }^{5}\right)$ y me dejó aquí tirado, me mandó la plata para hacer las vueltas y me dio uno que otro papel falso y ya" (ES_Harold_26)

Sin embargo, en los casos analizados la relación finaliza después de un corto tiempo de establecida, debido a la decepción frente a las expectativas que se habían idealizado hacia la otra persona. Esta situación pone al inmigrante en una condición de vulnerabilidad que "surge como consecuencia de la interacción social de los extranjeros que entran en un país con los "nacionales" (Bustamante, 1998, p. 8).

La vulnerabilidad de estos varones tiene su origen en la ausencia de redes sociales de apoyo, los cuales han llegado casi exclusivamente por gestiones de sus parejas en España. El inmigrante se encuentra sujeto a las condiciones que le imponga su pareja, por lo que al separarse su situación administrativa se vuelve irregular debido a que su entrada al país se realizó con un visado de turista con una duración definida, carece de todo tipo de apoyo familiar o social, de recursos económicos para subsistir, y se ven abocados al trabajo sexual en un entorno desfavorable.

\section{CONSIDERACIONES FINALES}

La migración internacional es un proceso complejo y dinámico que involucra todos los ámbitos de la vida personal, familiar y social de los individuos que inician este camino. La sexualidad no es ajena a dicho proceso, en especial cuando la migración viene inmersa en una serie de imaginarios, conceptos e ideas formadas por el intercambio de experiencias con otros individuos emigrados (o no) y que además se nutren de los medios de comunicación.

Estos elementos dan un nuevo sentido a las percepciones de hombres y mujeres sobre sus cuerpos

5 El scat es el término utilizado para referirse a la coprofilia, que consiste en la excitación sexual producida por las heces. Incluye la atracción por oler, saborear, tocar o ver el acto de defecar como un medio de excitación sexual y de placer. y su sexualidad, los cuales se redefinen y reconstruyen bajo parámetros nuevos y diferentes en los contextos migratorios. Es así como los valores migrantes entrevistados responden a realidades diversas y en las cuales toman mayor valor los elementos referidos a sus propios intereses, expectativas de vida, deseos y anhelos los cuales son expresados a través de sus prácticas sociales, sus relaciones con los otros, sus cuerpos y sus estéticas.

Todo esto surge como resultado de la eliminación o por lo menos la disminución de la influencia de los controles sociales y los limitantes impuestos por la heteronormatividad que se vive en el país de origen, de tal modo que el nuevo contexto social y sexual español promueve en los migrantes el acceso a un conjunto de expresiones, de relaciones y de formas de ser varón, homosexual o bisexual que se ajustan a los intereses personales del migrante.

Estas nuevas formas de ser y entender la sexualidad desde otros puntos de vista y desde la experiencia como migrante determina las prácticas sociales que finalmente establecerán el grado de integración de estos en la sociedad de acogida, todo ello en un proceso constante de transformación social y cultural que se inicia aun antes de emprender el viaje, pero que no modifica de manera categórica algunas expresiones afectivo-sexuales, en especial aquellas referidas a la pareja, la fidelidad y el amor.

La migración se constituye entonces en una forma de hacer frente a las restricciones que imponen ciertos contextos sociales y culturales y brinda a los varones homosexuales y bisexuales una nueva perspectiva de vida, en la cual sus familias y las redes sociales que potencian el proceso migratorio tienen una gran importancia.

Por un lado, la familia puede dar inicio al proceso migratorio buscando brindarles a sus miembros seguridad, confianza y el goce pleno de sus derechos y para esto reagrupan a sus hijos cuando son conocedores de su orientación sexual. Lo que pone de manifiesto además, los nuevos aprendizajes adquiridos por los migrantes en el país de destino que actúan como remesas sociales y pueden originar cambios en el país de origen.

Por otro lado, las redes sociales constituidas por amigos aseguran la acogida de los migrantes 
en España, en los casos en los cuales estos varones desean permanecer en el anonimato y disminuir los controles sociales que pueden ejercer sus familiares, por tanto ambas redes se complementan.

La migración sexual es un proceso complejo que debe analizarse de forma integral vinculando aspectos sociales, culturales, económicos y políticos ya que en algunos casos la relación entre orientación sexual 0 identidad de género y el proceso migratorio no es evidente. Esto se debe a que algunos proyectos migratorios pueden estar justificados bajo otro tipo de motivaciones que, sin embargo, son parte integral de un proceso migratorio estructurado en torno a la identidad sexual de quien migra.

Existen varias estrategias dentro de la migración sexual que responden a las necesidades particulares del migrante, algunos de estos varones migran en busca de espacios sociales donde sean aceptados y respetados, otros han sido reagrupados por sus familias quienes desean brindarles seguridad y finalmente hay quienes migran con sus pareja 0 al encuentro de estas en el país de destino, para emprender una vida en familia.

La relación entre migración y la sexualidad debe plantearse desde una visión integral que contribuya a comprender y analizar las dinámicas sociales tanto a la sociedad de origen como en el país de acogida.

\section{BIBLIOGRAFÍA}

Baile, J. (2008). Estudiando la homosexualidad. Teoría e investigación. Ediciones Pirámide. Madrid, España.

Bustamante, J. (1998). Marco conceptual de referencia sobre la vulnerabilidad de los migrantes como sujetos de derechos humanos, Consejo económico y social de las Naciones Unidas. Ginebra, Suiza, (en línea) http://www.unhchr.ch/ HURIDOCDA/HURIDOCA.NSF/2E8212C54BAB43 B9C1256991004E96D9/1F9A9533600C4DDE8 02566C00053C17D?OPENDOCUMENT, consultado el 29 de febrero de 2012.

Butler, J. (2007). El género en disputa, el feminismo y la subversión de la identidad. Paidós Studio. Barcelona, España.
Cantú, L. J., (2009). The sexuality of migration: Borber crossings and mexican inmigrant men. New York University Press.

Carrillo, H., Fontdevila, J., Brown, J., y Gómez, W. (2008). Fronteras del riesgo. Contextos sexuales y restos para la prevención del VIH entre inmigrantes mexicanos gay y bisexuales. Gender for research on gender and sexuality, San Francisco State University.

Dhoest, A. (2016). Media, visibility and sexual identity among gay men with a migration background. Sexualities, vol. 19, Issue, 4, 2016, pp. 412-431. University of Antwerp, Belgium.

Fajardo, M.; Patiño, M. y Patiño, C. (2008). Estudios actuales sobre aculturación y salud mental en inmigrantes: revisión y perspectivas. Revista iberoamericana de psicología: ciencia y tecnología. Núm. 1, pp. 39-50.

Falconí, T. D. (2015). De migraciones y homosexualidades latinoamericanas. Viaje, pérdida e imperceptibilidad en las novelas Los caminos a Roma y Años de indulgencia de Fernando Vallejo. Revista Impossibilia. Núm. 9, pp. 28-48.

García, F. J. (2013). Patrones de migración en Colombia desde la perspectiva de la teoría de redes. Cuadernos de Economía. Núm. 59, vol. 32, pp. 339-364.

Gallardo, L. y Escolano, F. (2009). Informe Diversidad Afectivo-Sexual en la Formación de Docentes. Evaluación de Contenidos LGTB en la Facultad de C.C.E.E. de Málaga. Guarnizo, L. (2004). Assimilation and transnationalism: Determinants of transnational political action among comtemporany migrants. Biblioteca virtual del Banco de la República. Bogotá, Colombia.

Guasch, 0. (1993). Para una sociología de la sexualidad. Revista Española de Investigaciones Sociológicas, núm. 64.

Guasch, 0. (1995). La sociedad rosa. Editorial Anagrama, Barcelona, España.

Instituto Nacional de Estadística (INE). (2007). Documentos de Trabajo 2/08. Encuesta Nacional de Inmigrantes 2007, Madrid, España.

Jolly, S. y Reeves, H. (2005), Género y migración: Informe general,Institute of Development Studies, University of Sussex, Brighton, Reino Unido. 
Khoudour-Castéras, D. (2007). ¿Por qué emigran Ios colombianos? Un análisis departamental basado en el censo de 2005. Revista de Economía Institucional, vol. 9, núm. 19, Primer semestre/2007, pp. 255-271.

Lomnitz, Larissa. (1981). Cómo sobreviven las marginadas. México, Siglo XXI.

Massey, D., S. Arango, Graeme, J., Kouaouci, H., PeIlegrino, A. y Taylor, E. (2008). Teorías de migración internacional: una revisión y aproximación. Revista REDCE, n. ${ }^{0}$ 10: 435-478.

Massey, D. Durand, J. y Nolan J. Malone. (2009). Detrás de la trama. Políticas migratorias entre México y Estados Unidos. Colección América Latina y el Nuevo orden Mundial. México: Miguel Ángel Porrua, UAZ. Cámara de Diputados LIX Legislatura.

Mead, G. H. (1991). La génesis del self y el control social. Revista REIS, julio-septiembre, núm. 55, pp. 165-186.

Orellana, Gilda. (2014). Somos lo que nadie te contó. En Falconí Trávez, Diego (ed.). "Me fui a volver". Narrativas, autorías y lecturas teorizadas de las migraciones ecuatorianas. pp. 43-59.

Organización Panamericana de la Salud, Organización Mundial de la Salud, (2000). Promoción de la salud sexual: Recomendaciones para la acción, Actas de una reunión de consulta convocada por la OPS, OMS y la Asociación Mundial de Sexología (WAS)", mayo 19-22, Antigua Guatemala, Guatemala.

Ramírez, C., Zuluaga, M. y Perilla, C. (2010). Perfil migratorio de Colombia. Organización Internacional de las Migraciones, Bogotá, DC, Colombia.

Restrepo, P. J. (2013). Sexualidades migrantes: La experiencia migratoria de los hombres homosexuales y bisexuales colombianos en España",
Revista Latinoamericana de Estudios sobre Cuerpos, Emociones y Sociedades. (en línea) http://www.relaces.com.ar/index.php/relaces/ article/viewArticle/204.

Restrepo, P. J. (2015). Migración sexual. Estrategias migratorias de los varones homosexuales y bisexuales colombianos en Sexualidades, Represión, Resistencia y Cotidianidades. Valcuende del Río, J. M., Vásquez, P. y Marco, M. (Coords.). Editorial Aconcagua Libros, Sevilla, España.

Restrepo, P. J. (2016). Análisis comparativo de las percepciones sobre VIH/SIDA de varones homosexuales y bisexuales colombianos, con experiencia migratoria 0 sin la misma. Revista de Salud Pública, vol. 18, núm. 1, febrero, 2016, pp. 2636. Universidad Nacional de Colombia, Facultad de Medicina, Instituto de Salud Pública.

Toro. J., López, M. y Nieves, K. (2012). Sexualidades migrantes: La emigración de hombres dominicanos gay. Revista Caribbean Studies, vol. 40, núm. 1, enero-junio, 2012, pp. 59-80. Instituto de Estudios del Caribe, San Juan, Puerto Rico.

Spargo, T. (2007). Foucault y la teoría Queer. Editorial Gedisa, 2. ${ }^{a}$ Edición, Barcelona, España.

Viveros, M. (2002). De quebradores y cumplidores: sobre hombres, masculinidades y relaciones de género en Colombia. CES, Universidad Nacional de Colombia.

Weeks, R. J. (1990). Sociología de la población, introducción a los conceptos y cuestiones básicas. Alianza Editorial, Madrid, España.

Wimark, T. (2016). Migration motives of gay men in the new acceptance era: a cohort study from Malmö, Sweden. Social and Cultural Geography. vol. 17, Issue 5, 3 July 2016, pp. 605-622. Department of Human Geography, Stockholm University, Stockholm, Sweden. 